\title{
Importancia del acabado y pulido en restauraciones directas de resina compuesta en piezas dentarias anteriores. Reporte de Caso
}

\author{
Importance of finishing and polishing direct restorations of resin composite in anterior teeth. Case \\ Report
}

César Lamas-Lara ${ }^{1, b}$, Sergio Alvarado-Menacho ${ }^{2, a, b}$, Giselle Angulo de la Vega ${ }^{2, a}$

\section{RESUMEN}

Las restauraciones directas con resina compuesta realizadas en nuestros pacientes requieren mucha pericia por parte del operador, y a pesar que, a pesar que en muchos de los casos cumplimos con los protocolos adhesivos requeridos y la inserción correcta del material en la preparación; sin embargo, luego de realizar todo el proceso restaurador, dedicamos muy poco tiempo al acabado y pulido siendo este paso fundamental para lograr el éxito. Muchos no logramos obtener resultados satisfactorios al utilizar un sistema de acabado y pulido porque no sabemos utilizarlo adecuadamente, no sabemos cuánto tiempo dura el proceso, cual es la secuencia correcta o que aislante térmico utilizar. En el presente artículo se detalla la resolución de un caso clínico a través de un método de acabado y pulido de restauraciones directas sobre resina compuesta en el sector anterior. Los resultados obtenidos fueron satisfactorios ya que se pudo mejorar las restauraciones devolviendo la naturalidad, estética y función perdida preservando la vitalidad pulpar a través de protocolos conservadores. Concluimos, que el proceso de acabado y pulido de las restauraciones con resina compuesta es fundamental para garantizar el éxito del tratamiento, debemos de conocer a cabalidad el sistema de pulido para no injuriar la pulpa dental y así no perjudicar su vitalidad.

PALABRAS CLAVE: Resinas compuestas, oxido de aluminio, pulpitis, estética dental. (DeCS, Bireme)

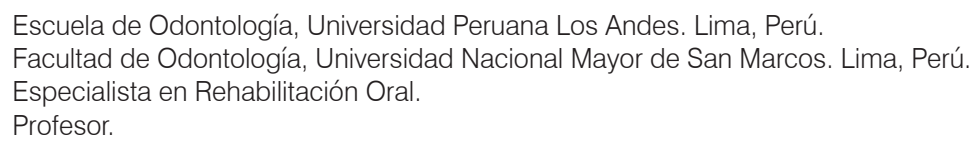




\section{SUMMARY}

Direct composite resin restorations performed in our patients require much expertise by the operator and in many cases we comply with the required adhesive protocols and the correct inclusion of the material in the preparation, but after the restorative process we dedicate very little time to the finishing touch and polish this being a fundamental step for success. Many fail to obtain satisfactory results by using a system of finishing and polishing because we don't use it properly, do not know how long the process takes, or which is the correct sequence or insulation to use. This article details the resolution of a clinical case through a method of finishing and polishing of restorations on direct composite resin in the anterior teeth. The results obtained were satisfactory since they failed to improve restorations returning natural, aesthetic and lost function preserving the vitality pulp through conservative protocols. We conclude that the process of finishing and polishing our composite resin restorations was crucial to ensure the success of our treatments, but we must know perfectly and adequately the system used so as not to injure the dental pulp and thus not jeopardize its vitality.

\section{KEYWORDS: Composite resins, aluminum oxide, pulpitis, dental aesthetic. (MeSH, NLM)}

\section{INTRODUCCIÓN}

Cuando realizamos una restauración con resina compuesta nos preocupamos bastante por realizar la correcta preparación de la cavidad, un correcto protocolo adhesivo y una correcta inserción del material, pero en muchos casos descuidamos realizar adecuadamente el acabado y pulido de la restauración y a veces no consideramos nuestros errores en esta parte fundamental como la causa de fracaso.

Debemos de considerar como objetivo primordial realizar la conservación de las superficies de las restauraciones; es decir, conservarlas sin manchas o acúmulos de placa bacteriana y esto lo lograremos reduciendo las superficies ásperas, márgenes inadecuadamente acabados y retirando los excesos del material sobre todo en los bordes cerca a las encías ya que esto es un factor para la aparición de caries secundarias (1-3).

En la actualidad hay muchos intentos por reducir la cantidad de instrumentos relacionados con la etapa de acabado y pulido, es decir un solo instrumento para todos los pasos, pero estos intentos todavía no logran el consenso entre los investigadores para poder decir que reemplazan toda nuestra secuencia tradicional. Actualmente utilizamos sistemas de granulación progresiva es decir usamos instrumentos abrasivos con granulación gruesa inicialmente y terminamos con una granulación más fina $(4,5)$.

Al realizar el acabado de la restauración la parte superficial es removida por una serie de cortes y este procedimiento deja una superficie áspera que requiere un pulido. Esta superficie se considera pulida cuando esas imperfecciones de la superficie pasen inadvertidas al ojo humano observándose tersa y brillante. Valores superiores a los $15 \mu \mathrm{m}$ son considerados desagradables por el sistema nervioso central, aunque estudios recientes demuestran que estos valores serían menores ya que valores por encima de $0.3 \mu \mathrm{m}$ llegan a ser percibidos (6-8).

La rugosidad superficial de las resinas compuestas depende del tamaño de las partículas de relleno que presenten y de la técnica de pulido utilizada. De acuerdo a la clasificación actual de resinas compuestas estamos utilizando resinas nanohibridas y de nanorelleno que presentan buenas propiedades ópticas como una adecuada retención de brillo y textura superficial (9).

El objetivo del presente caso fue devolver al paciente una adecuada estética y función masticatoria con el uso de materiales restauradores directos y realizar una adecuada secuencia de acabado y pulido evitando producir daño a las estructuras dentarias remanentes.

\section{REPORTE DEL CASO}

Paciente de sexo femenino de 30 años de edad que acude a la consulta clínica expresando el deseo de realizar el cambio de restauraciones antiguas realizadas hace 10 años. 
Al examinar a la paciente se pueden apreciar restauraciones con resina compuesta a nivel de las piezas $12(\mathrm{M}), 11(\mathrm{MI}), 21(\mathrm{MI})$ y $22(\mathrm{M})$, Figura 1.

\section{Diagnóstico}

\section{Del estado de salud general:}

Paciente con buen estado de salud general, sin riesgo sistémico al tratamiento estomatológico.

\section{Del estado de salud estomatológico:}

Paciente con presencia de restauración defectuosa en las piezas $12(\mathrm{M}), 11(\mathrm{MI}), 21$ (MI) y $22(\mathrm{M})$.

\section{Plan de Tratamiento}

1. Fisioterapia oral, enseñanza de la técnica de cepillado, uso de hilo dental y enjuague bucal.

2. Restauraciones directas con resina compuesta en piezas $12(\mathrm{M}), 11(\mathrm{MI}), 21(\mathrm{MI})$ y $22(\mathrm{M})$.

\section{Tratamiento}

Se procedió a realizar el retiro de la restauración de resina compuesta y la eliminación del tejido cariado con aislamiento absoluto.
Se decidió utilizar un sistema adhesivo de grabado total de dos pasos (Adper ${ }^{\mathrm{TM}}$ Single Bond 2 de $3 \mathrm{M}^{\mathrm{TM}}$ $\mathrm{ESPE}^{\mathrm{TM}}$ ). Se realizó la aplicación de ácido fosfórico al 37\% 15 segundos en dentina y 30 segundos en esmalte, posteriormente se lavó con abundante agua y se secó con papel absorbente sin resecar la dentina. Se aplicó el adhesivo de quinta generación frotándolo profusamente en la superficie de la preparación y se fotopolimerizó por 20 segundos. Posteriormente, se realizó la aplicación de una resina con nanotecnología (Filtek $^{\mathrm{TM}}$ Z350 XT de $3 \mathrm{M}^{\mathrm{TM}} \mathrm{ESPE}^{\mathrm{TM}}$ ) utilizando la técnica por capas naturales para lograr una mayor estética en el sector anterior (Figura 2).

Una vez realizada las restauraciones procedemos a realizar el acabado y el pulido utilizando discos de acetato con una cubierta de óxido de aluminio. Para reducir el calor por fricción se utilizó como medio de aislante térmico vaselina sólida y agua. Con el primer disco de granulación gruesa se realiza la reducción de los excesos del material, este disco debe de ser utilizado a baja velocidad (10 $000 \mathrm{rpm})$ de manera intermitente entre 15 a 20 segundos, realizando movimientos unidireccionales con suave presión y evitando el contacto del borde interno metálico para evitar rayar la restauración (Figura 3).

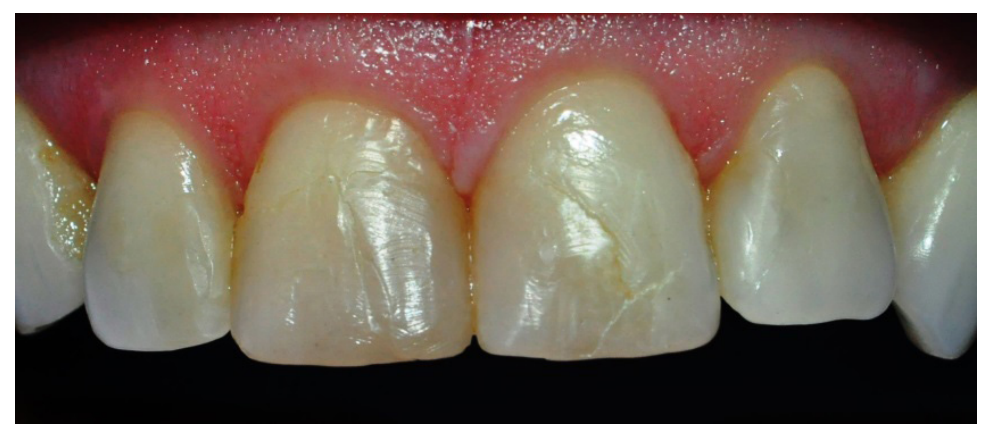

Figura 1. Vista inicial. Presencia de restauraciones defectuosas.

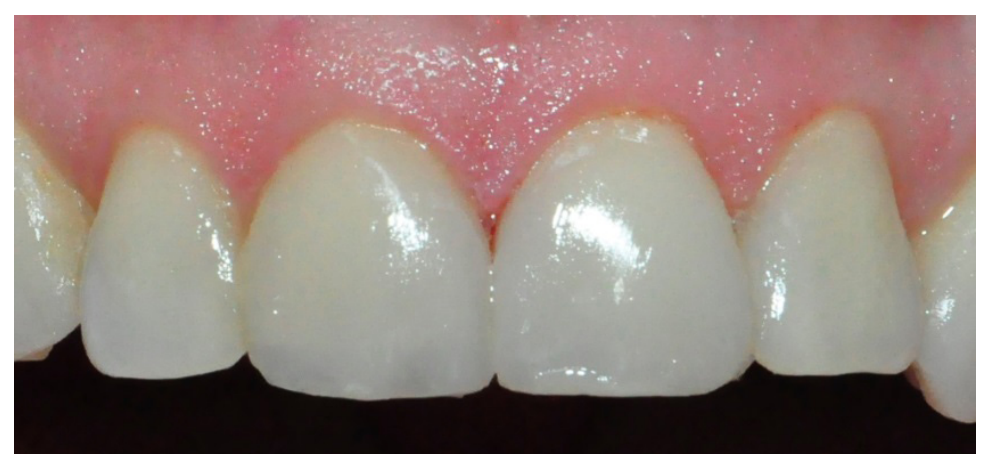

Figura 2. Vista post restauración. Restauraciones cubiertas con glicerina líquida. 


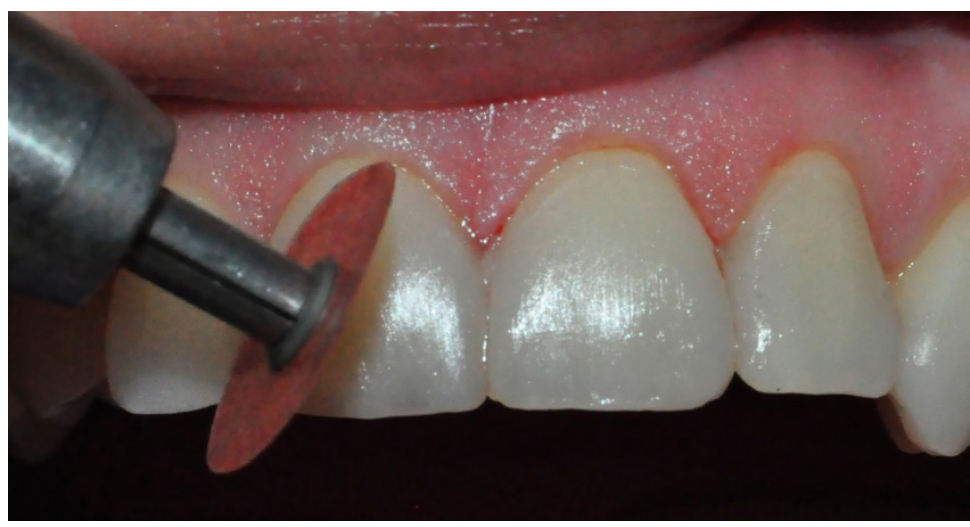

Figura 3. Primer disco: con oxido de aluminio.

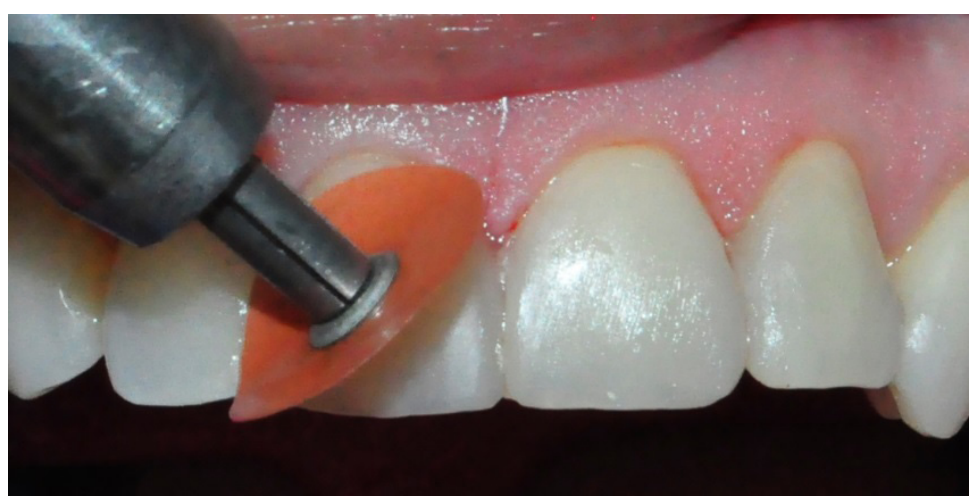

Figura 4. Segundo disco: con óxido de aluminio.

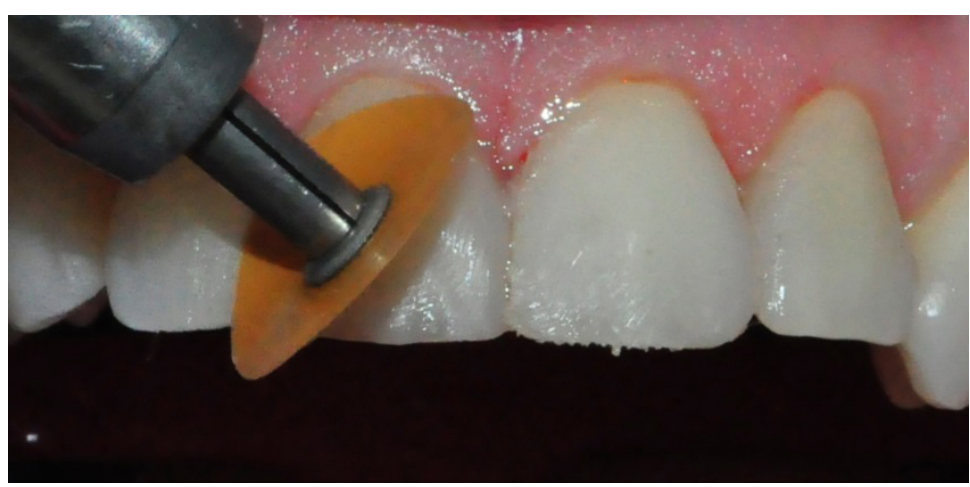

Figura 5. Tercer disco: con óxido de aluminio.

El segundo disco de granulación media se utiliza a $10000 \mathrm{rpm}$ de manera intermitente por 15 a 20 segundos para darle el contorneado final, realizando movimientos unidireccionales desde cervical a incisal y con ligera presión (Figura 4). El tercer disco de granulación fina se utiliza para darle el acabado de la resina compuesta a una velocidad de hasta 10 $000 \mathrm{rpm}$ por 15 a 20 segundos realizando los mismos movimientos que los discos anteriores (Figura 5). Y el último disco de granulación ultrafina es utilizado para realizar el pulido final a una velocidad de hasta $10000 \mathrm{rpm}$ por 15 a 20 segundos (Figura 6). Recordar que se debe aplicar agua sobre la superficie a pulir con el fin de evitar el aumento de temperatura.

Una vez terminado la secuencia de discos, se complementa el pulido con el uso de una escobilla de pelo de cabra a baja velocidad (10 $000 \mathrm{rpm})$ por un periodo de 20 segundos con vaselina solida sobre las superficies (Figura 7). 


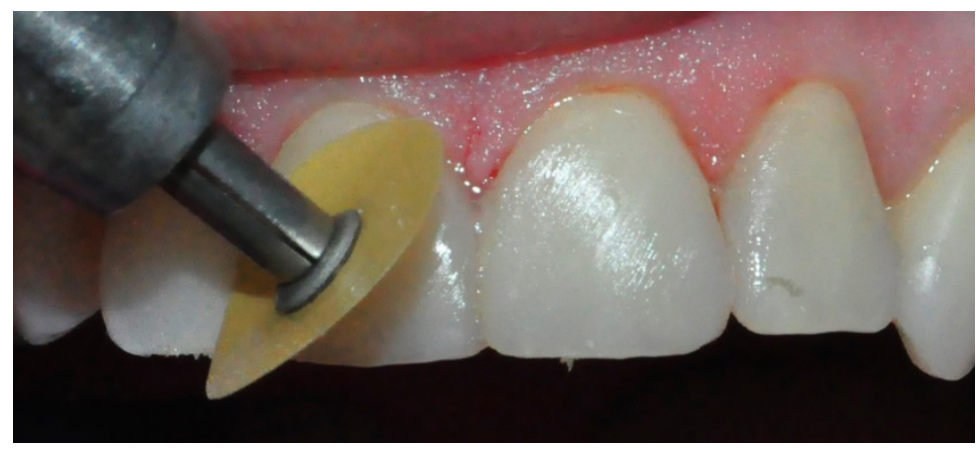

Figura 6. Cuarto disco: con óxido de aluminio.

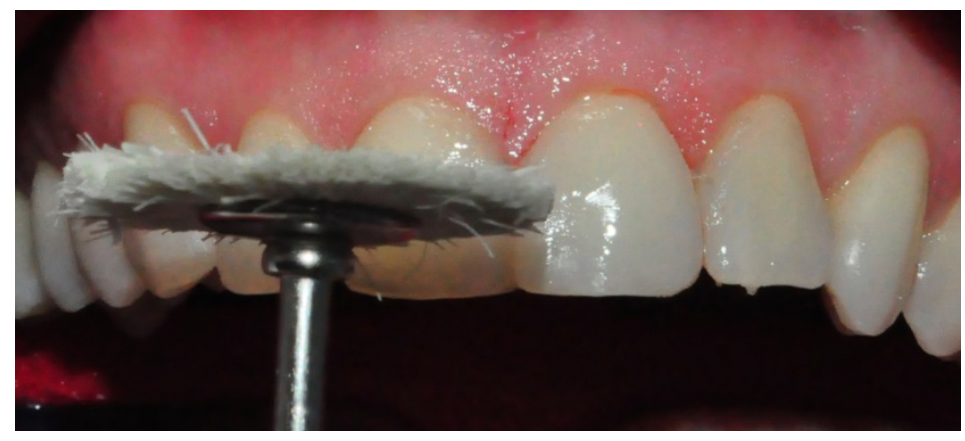

Figura 7. Uso de la escobilla de pelo de cabra.

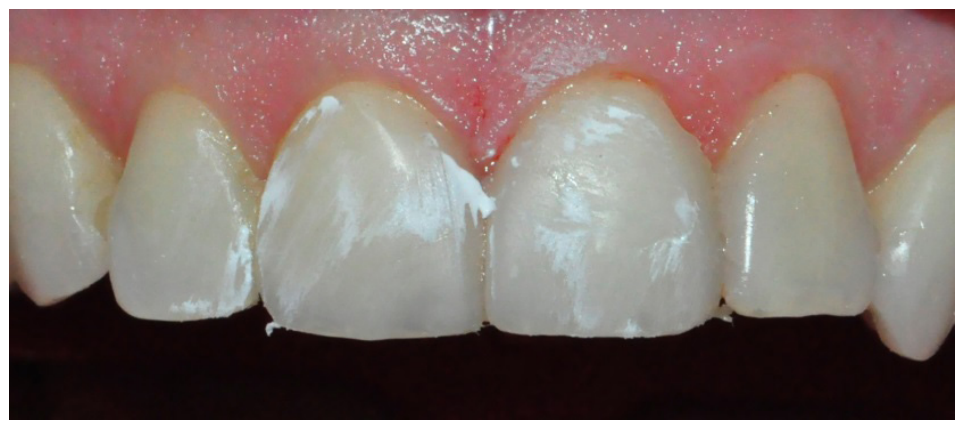

Figura 8. Aplicación de una pasta de pulido.

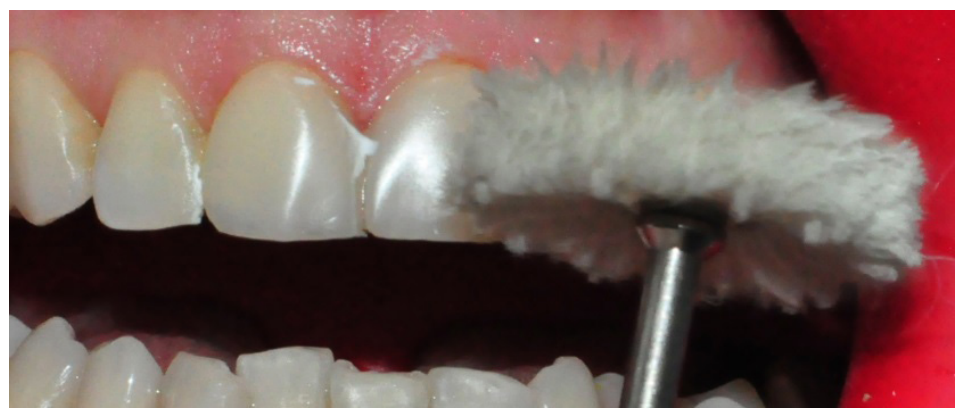

Figura 9. Uso de un fieltro con la pasta de pulido.

Una vez realizado este paso aplicar una pasta de pulido, utilizando un fieltro a $10000 \mathrm{rpm}$ por un periodo de 20 segundos, primero seco y luego mojando las superficies a pulir (Figura 8 y 9 ). Se retiran los restos que se acumulan en las superficies con una escobilla de profilaxis y vaselina sólida, una vez realizada la secuencia, evaluamos los resultados obtenidos (Figura 10). 


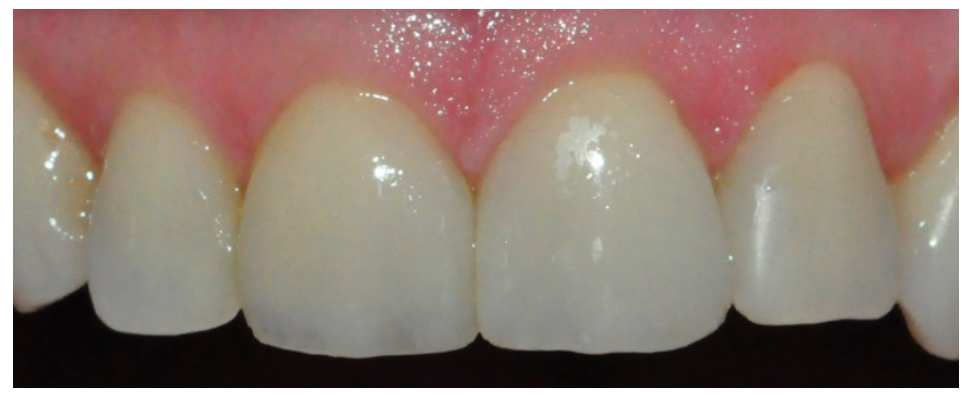

Figura 10. Vista final de la restauración.

\section{Resultados}

Los resultados obtenidos fueron bastante buenos para la paciente, se logró una morfología satisfactoria reduciendo la rugosidad superficial de las resinas compuestas e igualando el brillo de las mismas a las piezas dentarias sanas, devolviéndole así la función y la estética perdida.

\section{DISCUSIÓN}

Al realizar restauraciones directas con resina compuesta son muchas las consideraciones a tomar en cuenta para no ocasionar un daño pulpar y una de ellas es no generar demasiado calor friccional al realizar el acabado y pulido ya que podemos ocasionar daño pulpar irreversible. Entonces, debemos considerar el tiempo de contacto de la punta diamantada, caucho, disco, escobilla o fieltro sobre la superficie dentaria, la velocidad de rotación del micromotor y el medio de aislante térmico entre el instrumento de pulido y la superficie dentaria como factores primordiales. Estudios demuestran que el uso de aislante térmico no afecta la reducción de la rugosidad superficial de la resina compuesta; por consiguiente, no afecta el pulido de la restauración (10-14).

Estudios demuestran que si se realiza el pulido inmediato no lograremos reducir adecuadamente la rugosidad superficial, esto se puede deber a que el grado de conversión de las resinas compuestas activadas con las lámparas de luz halógena bordea el $70 \%$, lo que quiere decir que hay $30 \%$ de resina compuesta sin polimerizar; y si realizamos el pulido en la misma cita, esa matriz de resina suave se esparce por la superficie dando un efecto mate de la misma, por lo que se recomienda esperar una cita posterior para realizar el pulido final de la restauración $(12,15)$.

\section{CONCLUSIONES}

Realizar el acabado y pulido de las restauraciones directas de resinas compuesta es una parte importante de nuestro procedimiento restaurador ya que así estamos garantizando la longevidad de la misma.

\section{Correspondencia:}

César Lamas Lara

Santa Honorata 415, Urb. Pando, 3era Etapa, San Miguel. Lima, Perú.

Correo electrónico: cesar2579@hotmail.com

\section{REFERENCIAS BIBLIOGRÁFICAS}

1. Bollen CML, Lambrechts P, Quirynem M. Comparison of surface roughness of oral hard materials to the threshold surface roughness for bacterial plaque retention: a review of the literature. Dent Mater. 1997; 13 (4):258-69.

2. Jung M. Finishing and polishing of a hybrid composite and a heat-pressed glass ceramic. Oper Dent. 2002; 27(2):175-83.

3. Neme AL, Frazier KB, Roeder LB, Debner TL. Effect of prophylactic polishing protocols on the surface roughness of esthetic restorative materials. Oper Dent. 2002; 27(1):50-8.

4. Türkün LS. Effect of re-use of a disposable micropolisher on the surface of a microhybrid resin composite. Am J Dent. 2004; 17(4):279-82.

5. Marigo L, Rizzi M, La Torre G, Rumi G. 3-D Surface profile analysis: different finishing methods for resin composites. Oper Dent. 2001; 26(6):562-8.

6. Setcos J, Tarim B, Suzuki S. Surface finish produced on composites by new polishing systems. Quintessence Int. 1999; 30(3):169-73.

7. Jones $\mathrm{C}$, Billington R, Pearson G. The 'in vivo' perception or roughness of restorations. Br Dent J. 2004; 196(1): 42-5. 
8. Kamonkhantikul K, et al. Polishing and toothbrushing alters the surface roughness and gloss of composite resins Dent Mater J 2014; 33(5):599-606.

9. Herrgott A, Ziemiecki T, Dennison J. An evaluation of different composite resin system finished with various abrasives. J Am Dent Assoc. 1989; 119 (6):72932.

10. Senawonqse P, Ponqprueksa P. Surface roughness of nanofill and nanohybrid resin composites after polishing and brushing. J Esthet Restor Dent. 2007; 19(5):265-75.

11. Jung O. Is surface roughness of resin composites affected by operator's performance? Am J Dent. 2008; 21(1):3-6.

12. Da Silva JM, da Rocha DM, Travassos AC, Fernandez W Jr., Rodriguez JR. Effect of different finishing time on surface roughness and maintenance of polish in nanoparticle and microhybrid compisite resins. Eur $\mathrm{J}$
Esthet Dent. 2010; 5(3):288-98

13. MirzaKoucheki P, Daneshpour N, Zare M. The effect of different polishing methods and composite resin thickness on temperature rise of composite restorative materials. JIDA 2012; 24(4):237-43.

14. Lavin N, Toro U. Cuantificación de la generación térmica en resina compuesta sometida a tres sistemas de pulido in vitro. Int J Odontostomat. 2012; 6(3):26773.

15. Suarez R, Lozano F. Comparación de la dureza superficial de resinas de nanotecnología, según el momento del pulido: in vitro. Rev Estomatol Herediana. 2014; 24(1):11-6.

Recibido: 07/04/2015

Aceptado: 5/05/2015 